\title{
Correction to: Decoding COVID-19 pneumonia: comparison of deep learning and radiomics $\mathrm{CT}$ image signatures
}

\author{
Hongmei Wang ${ }^{1} \cdot$ Lu Wang $^{2} \cdot$ Edward H. Lee ${ }^{3} \cdot$ Jimmy Zheng ${ }^{3} \cdot$ Wei Zhang ${ }^{4} \cdot$ Safwan Halabi ${ }^{3} \cdot$ Chunlei Liu $^{5,6}$. \\ Kexue Deng ${ }^{1} \cdot$ Jiangdian Song ${ }^{7,8} \cdot$ Kristen W. Yeom $^{3}$
}

Published online: 3 March 2021

(C) Springer-Verlag GmbH Germany, part of Springer Nature 2021

\section{Correction to: Eur J Nucl Med Mol Imaging https://doi.org/10.1007/s00259-020-05075-4}

There was a mistake in the original article. One of the affiliations listed for Dr. Jiangdian Song is incorrect. The correct affiliation should have been:

${ }^{7}$ College of Medical Informatics, China Medical University, Shenyang, Liaoning, 110122, China

instead of

${ }^{7}$ Northeastern University, Shenyang 110819, Liaoning, China.

The original article has been corrected.

Publisher's note Springer Nature remains neutral with regard to jurisdictional claims in published maps and institutional affiliations.

This article is part of the Topical Collection on Erratum

The online version of the original article can be found at https://oi.org/ 10.1007/s00259-020-05075-4

Jiangdian Song

song.jd0910@gmail.com

1 Department of Radiology, The First Affiliated Hospital of University of Science and Technology of China (USTC), Division of Life Sciences and Medicine, USTC, Hefei 230036, Anhui, China

2 School of Medical Informatics, China Medical University, Shenyang 110122, Liaoning, China

3 Department of Radiology, School of Medicine Stanford University, 725 Welch Rd MC 5654, Palo Alto, CA 94305, USA
4 Department of Radiology, The Lu'an Affiliated Hospital, Anhui Medical University, Luan 237000, Anhui, China

5 Department of Electrical Engineering and Computer Sciences, University of California, Berkeley, CA 94720, USA

6 Helen Wills Neuroscience Institute, University of California, Berkeley, CA 94720, USA

7 College of Medical Informatics, China Medical University, Shenyang 110122, Liaoning, China

8 Department of Radiology, School of Medicine Stanford University, 1201 Welch Rd Lucas Center PS055, Stanford, CA 94305, USA 